\title{
A Quantification System of Needle Visibility in B-mode Ultrasound with Linear and Curved Transducer
}

\author{
Hesty Susanti, Arkanty Septyvergy, Suprijanto, and Deddy Kurniadi
}

\begin{abstract}
Apart from its usefulness in interventional procedure, ultrasound-guided needle insertion has also crucial problem in terms of needle visibility. The inconsistency of needle visibility is needed to be quantified to evaluate the significance of possible technical factors, e.g., imaging strategies, needle type, and needle-transducer relative position. Needle visibility quantification is important as an initial step before further investigation about fundamental physics behind it and further development of needle visibility enhancement.

20G, $150 \mathrm{~mm}$ spinal needle inserted in degassed water phantom is imaged with B-mode Flex Focus $800 \mathrm{BK}-$ Medical using $12 \mathrm{MHz}$ linear transducer and $6 \mathrm{MHz}$ curved transducer. The insertion angles are varied between $15^{\circ}-70^{\circ}$. The quantified visibility representing each needle position are combined into a comprehensive visibility map covering the whole insertion area. It is also evaluated based on insertion length.

The results suggest that both linear and curved transducer, for all insertion angles, the distributions of needle visibility have similar pattern and they are not affected by the insertion length. Practically, this applied method of visibility quantification can be used as specific reference and to predict the distribution of needle visibility limited by the specification of needle and ultrasound system, i.e., range of transducer's frequency and needle's size.
\end{abstract}

Keywords-interventional procedure, needle visibility, ultrasound, visibility quantification.

\section{INTRODUCTION}

$\mathrm{N}$ EEDLE insertion procedure with ultrasound guiding is considered useful because of some advantages of ultrasound, e.g., the absence of radiation risk, compact size of transducer and equipment, and the flexibility of imaging plane.

This work was supported by Indonesia Endowment Fund for Education (LPDP) and the Ministry of Research, Technology and Higher Education of Republic of Indonesia (competency-based research funding).

Hesty Susanti is Ph.D Candidate at Department of Engineering Physics, Bandung Institute of Technology, Indonesia

Arkanty Septyvergy is alumnus of Postgraduate Program (Master) of Instrumentation and Control, Bandung Institute of Technology, Indonesia

Suprijanto is Associate Professor in Instrumentation and Control Research Group, Bandung Institute of Technology, Indonesia

Deddy Kurniadi is Full Professor in Instrumentation and Control Research Group, Bandung Institute of Technology, Indonesia
At the same time, this interventional procedure has disadvantage in terms of poor quality and inconsistency of needle visibility. Needle visibility is one of crucial factors that affects the performance of needle insertion.

In the previous studies, the inconsistent and poor needle visibility is encountered within various range of ultrasound frequency and incident angle of ultrasound beam (Chin et al. 2008, de Jong et al. 2018, Dencks et al. 2014, Maecken et al. 2007, Susanti et al. 2018). In those cases, the frequency and incident angle affect needle visibility but no linear pattern has been found.

The inconsistency of needle visibility is needed to be quantified in order to evaluate the significance of possible technical factors that can affect it, e.g., imaging strategies, needle type, and relative position of the needle to the transducer. The quantification of needle visibility is important as an initial step before further investigation about fundamental physics behind it and eventually further development of needle visibility enhancement.

Previous studies related to needle visibility quantification are generally carried out with a scoring system by direct visual observation without applying any image processing method (Culp et al. 2000, Guo et al. 2012, Hebard et al. 2011, Karstrup et al. 2002, Marhofer et al. 2002). In this study, we propose a method of needle visibility evaluation by means of visibility leveling system which a further development of our previous study (Susanti et al. 2018). The leveling system is performed through B-mode ultrasound image processing with variation of insertion angles and depths. The quantified visibility that represents each needle position will be combined into a comprehensive visibility map covering the whole insertion area. It will also evaluated based on the insertion length.

In addition to previous motivation as an initial step before further investigation and needle visibility enhancement, in practical application, this applied method can be used as a specific reference for a physician before inserting the needle to ensure better needle placement to the region of interest.

\section{B-MODE ULTRASOUND MEASUREMENT}

Two B-mode ultrasound measurements are performed to evaluate needle visibility representing common clinical application, i.e. linear and curved transducer. 20G, $150 \mathrm{~mm}$ 
spinal needle (Uniplex Nano Line, Pajunk, Geislingen, Germany) is imaged with Flex Focus 800 BK-Medical (Brüel \& Kjær, Nærum, Denmark) with UGRA (Ultrasound-guided Regional Anesthesia) mode.

The first measurement is performed in frequency of 12 $\mathrm{MHz}$, maximum depth of $50 \mathrm{~mm}$, and variation of insertion angles of $15^{\circ}-60^{\circ}$ with linear BK-Medical transducer 8870 (6$18 \mathrm{MHz}$, Brüel \& Kjær, Nærum, Denmark). While the second measurement is conducted in frequency of $6 \mathrm{MHz}$, maximum depth of $90 \mathrm{~mm}$, and variation of insertion angles of $20^{\circ}-70^{\circ}$ with curved BK-Medical transducer 8802 (3-6 MHz, Brüel \& Kjær, Nærum, Denmark).

The measurements are performed with degassed demineralized water $\left(\rho=997 \mathrm{~kg} / \mathrm{m}^{3}\right)$ in a glass water chamber as background medium that is assumed to be homogeneous and anechoic. High frequency acoustic absorber Aptflex F28 (Precision Acoustics, Dorchester, Dorset, United Kingdom) is placed at the bottom of the tank to prevent the reflection from the tank's bottom wall.

In this measurement set up, the positioning system of needle and transducer can be adjusted so that the alignment of needle and transducer's face in elevational direction is in parallel with each other. The needle holder and transducer holder can position the needle so that it can be varied in the measurement range of insertion angle and depth. The measurement set up is shown in Fig. 1.
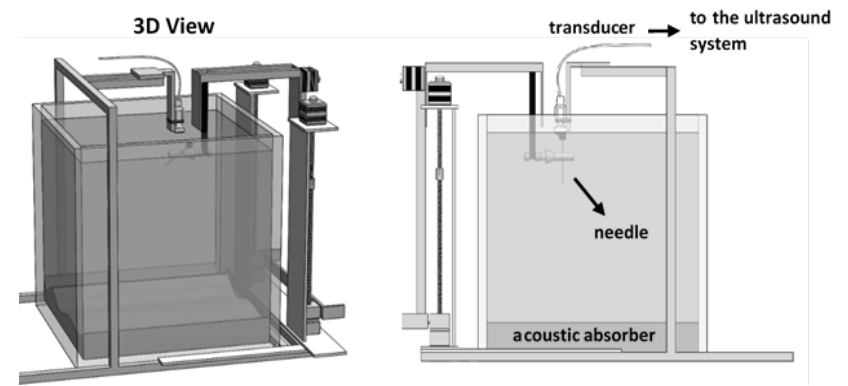

Fig. 1 the measurement set up that consists of water bath phantom as background medium, spinal needle as object medium, ultrasound system (with coresponding transducer), and needle-transducer positioning system

B-mode ultrasound images from the measurements will be processed to obtain representative visibility parameter. Insertion area consists of individual needle position marked by needle tip position. Needle tip positions represent the whole insertion area in representative insertion angles and depths.

The maps of needle tip positions for linear and curved transducer are shown in Fig. 2. Point $M$ is the initial point of insertion and $\mathbf{x}$ is the position of needle tip. The measurements are performed by varying the position of needle tip within the area of insertion. In this figure, the position of the transducer is above the area of insertion.

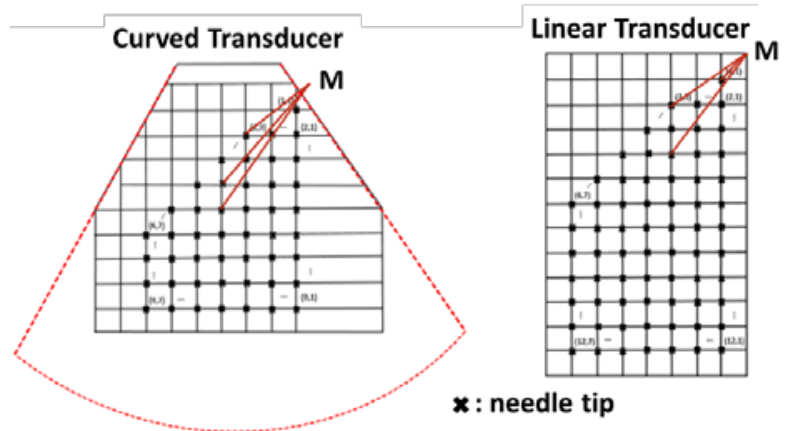

Fig. 2 the map of needle tip positions, $\mathrm{M}$ is initial point of insertion and the positions of needle tip in various angles and depths are marked by $\mathbf{x}$

III. Methodology

\section{A. The Region of Interest (ROI)}

To define the value of representative visibility parameter, the image is needed to be specified in a cer tain region of interest. In this case, the region of interest (ROI) is defined within the shaft of the needle excluding the tip. Fig. 3 shows the process of defining the ROI by cropping the area (green box) where the needle shaft appears in B-mode image. Then, the cropped image is processed furtherly by re-scaling the intensity in blueish-yellowish colored scale (0-255).

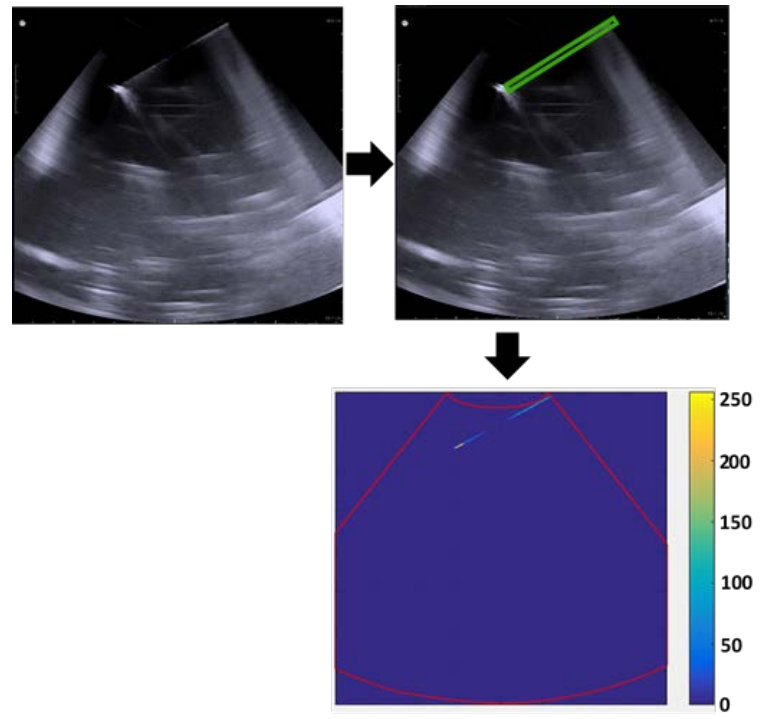

Fig. 3 defining the region of interest (ROI) within the shaft of the needle (green box)

\section{B. Visibility Parameter}

The defined visibility parameter represents the actual visibility in the region of interest, i.e., the actual intensity within the shaft of the needle. This parameter will be analyzed in two forms, i.e., graph of visibility distribution that represents the distribution of visibility based on the insertion length and visibility map that covers the whole area of insertion. 


\section{The Graph of Visibility Distribution}

Distribution pattern of visibility along the shaft of the needle will be analyzed based on the insertion length and insertion angle as illustrated in Fig. 4. Fig. 4 shows two needle positions with the same insertion angle $\left(35^{\circ}\right)$, but different insertion length, i.e., full insertion length $(30 \mathrm{~mm})$ and fractional (not full) insertion length $(12 \mathrm{~mm})$. Then, the intensity of the needle is extracted along the needle shaft and presented as the graph of visibility distribution.

Smoothing process is applied on each graph to define local maxima and local minima along the graph. The position of local maxima is calculated based on its percentage to the insertion length. For example, on the first graph in Fig. 4 ((a)), local maxima are in the positions of $25 \%, 55 \%$, and $78 \%$ of total insertion length of $12 \mathrm{~mm}$. While, on the second graph (Fig. 4 (b)), local maxima are in the positions of $17 \%, 45 \%$, and $82 \%$ of total insertion length of $30 \mathrm{~mm}$.

The position percentage of local maxima and local minima to the total length of the needle will be analyzed with statistical analyses, i.e., ANOVA (analysis of variance) for all insertion angles to evaluate their likeness.

For linear transducer, the insertion length for one insertion angle is classified into 2 lengths, i.e., full insertion length and one fractional (not full) insertion length. While for curved transducer, it c onsists of 3 lengths, i.e., full insertion length and 2 fractional (not full) insertion lengths.
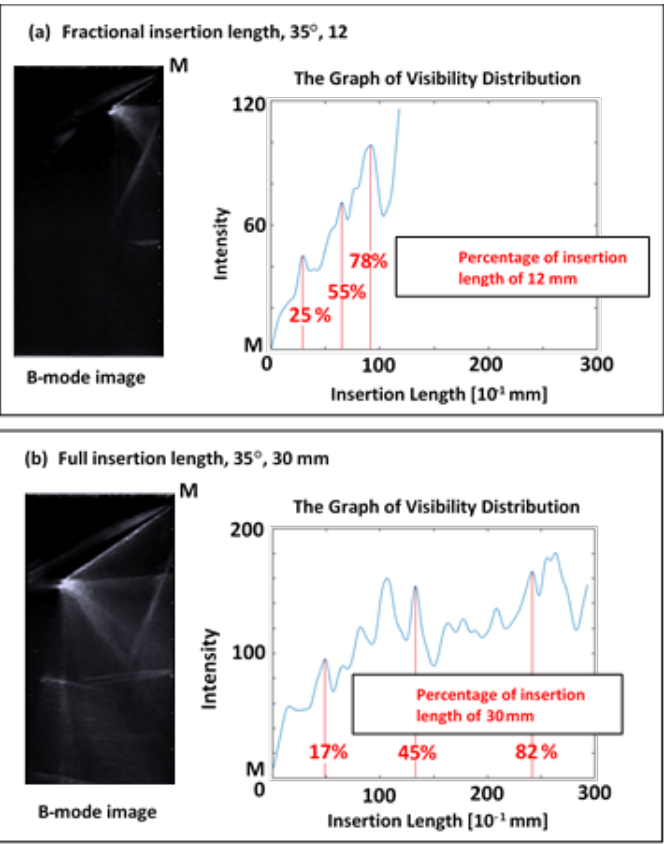

Fig. 4 the graph of visibility distribution extracted from two needle positions with same angle $\left(35^{\circ}\right)$ but different insertion length, i.e., fractional length $(12 \mathrm{~mm})$ and full length $(30 \mathrm{~mm})$

\section{The Visibility Map}

The configuration in the measurements is only possible to produce representative needle images based on the defined needle tip positions (Fig. 2). Those images are relatively limited and not continuous to cover the whole possible insertion angle and depth in the area of insertion. So, the area that are not covered by the measurement will be predicted with approximation methods, i.e., 1-dimensional interpolation. The pattern of visibility parameter that covers the whole area of insertion is defined as visibility map.

The visibility map is built in these following steps:

1) Needle images representing various insertion angles and depth are combined into one image that covers the whole area of insertion. In this case, the image is chosen from the longest insertion length for each insertion angle. (See Fig. 5).

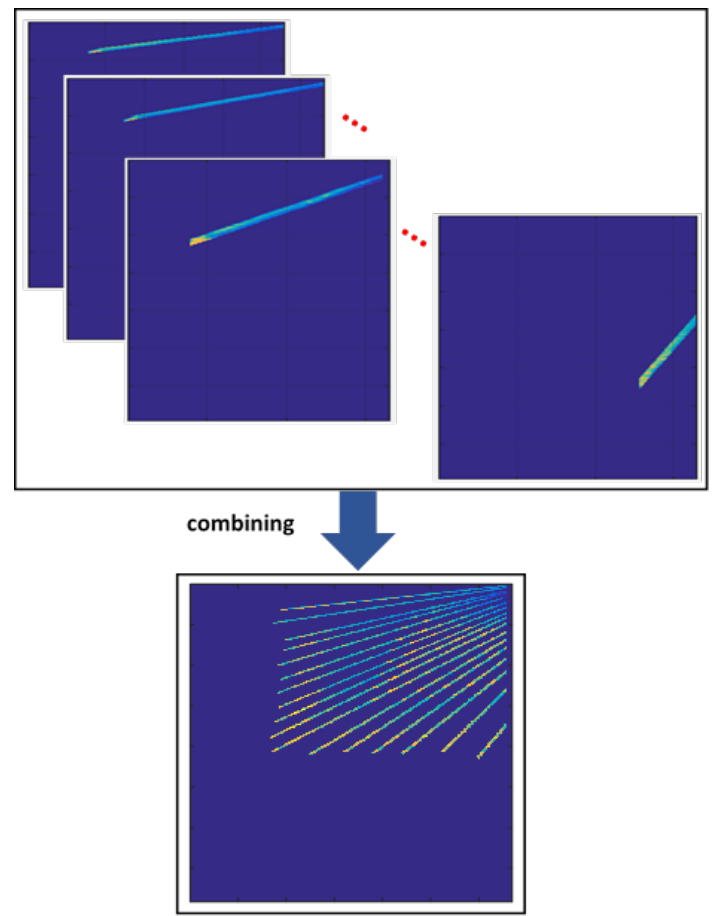

Fig. 5 combining needle images representing various insertion angles and depths into one image that covers the whole area of insertion angle

2) Combined image is cropped into narrower area where the approximation area will be focused. See Fig. 6 . 


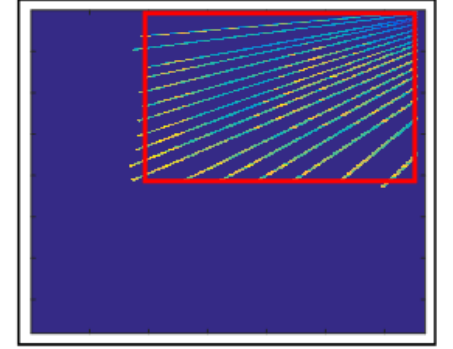

cropping

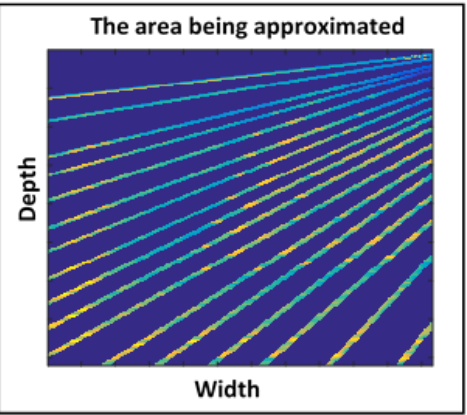

Fig. 6 cropping the combined image into narrower area

3) One-dimensional approximation will be conducted line per line vertically through 7 order polynomial interpolation (see Fig. 7). From the combined image (Fig. 7 (a)), it is extracted the intensity graph (Fig. 7 (b)). Here, the orange crosses show the value of needle intensities along vertical direction from the combined image, while, the blue line is approximated intensities by interpolating the orange crosses.

4) Finally, the intensity graphs are converted into colored scale and arranged line per line until forming 2-D intensity map in colored intensity scale. The intensity level represents gray-scales in 8-bit. This 2-D intensity map is called the visibility map (see fig. 7 (c)).

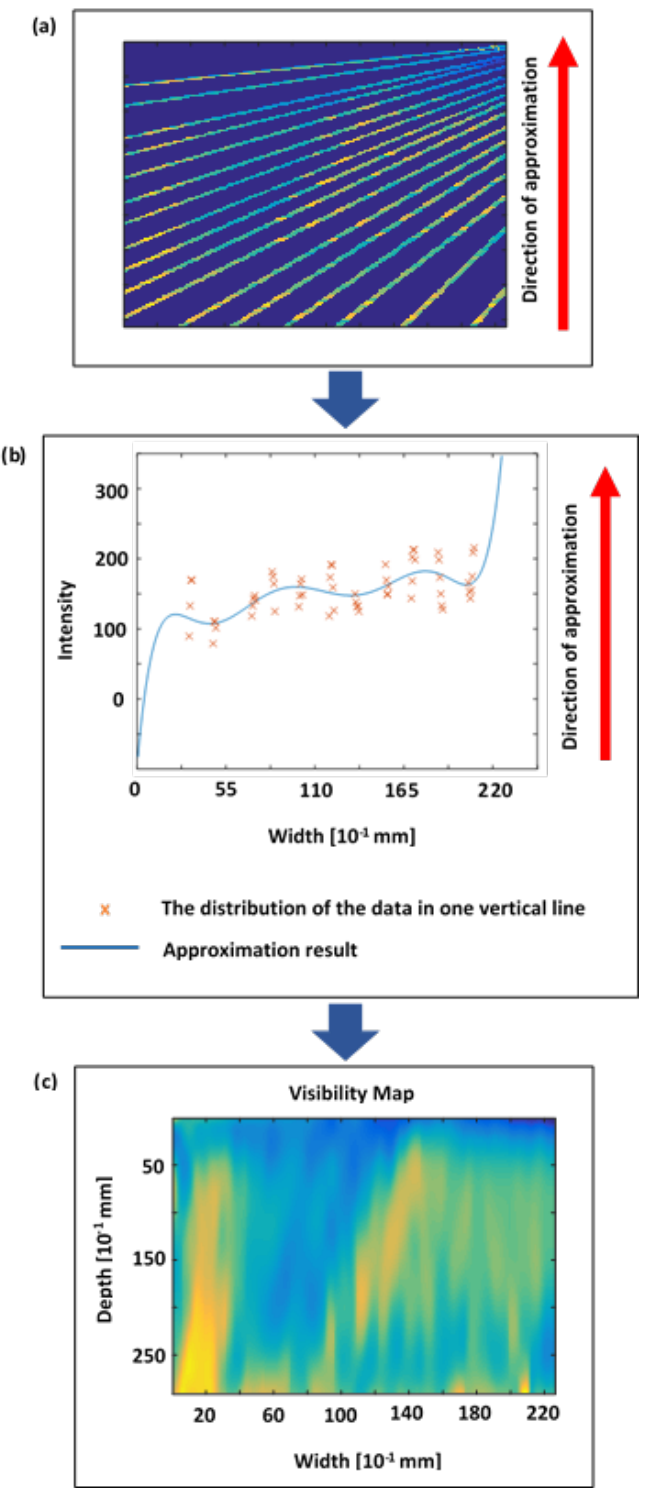

Fig. 7 one-dimensional interpolation on lateral (width) and axial (depth) direction, integrated into two-dimensional visibility map

In practical application, this visibility map can be cropped within a certain range of insertion angles. For example, in Fig. 8 , the visibility map is cropped between insertion angle of $25^{\circ}$ and $35^{\circ}$. The triangle-like segment within this focused range can be used to predict the visibility pattern more precisely within narrower area of the total insertion area. 


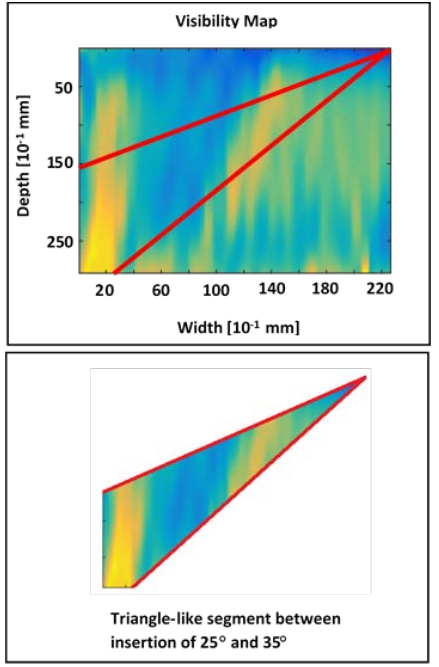

Fig. 8 cropping the area of the map into triangle-like segment between 2 evaluated insertion angles

\section{RESUlt AND DisCUSSION}

The visibility parameter will be analyzed in two forms, i.e., the graph of visibility distribution that represents the distribution of visibility based on the insertion length and visibility map that covers the whole area of insertion.

\section{A. The Graph of Visibility Distribution}

The graph of visibility distribution will be evaluated for 2 type of transducers, i.e., linear transducer $(12 \mathrm{MHz})$ and curved transducer $(6 \mathrm{MHz})$. This distribution pattern of visibility along the shaft of the needle will be analyzed based on the insertion length for each insertion angle.

The Graph of Visibility Distribution for Linear Transducer (12 $\mathrm{MHz})$

For linear transducer, the insertion angles are divided into 8 angles within the range of $20^{\circ}-60^{\circ}$. For each insertion angle, the insertion length is classified into 2 lengths, i.e., full insertion length and one fractional (not full) insertion length. The position percentage of local maxima and local minima to the total length of the needle will be analyzed with statistical analyses to evaluate their likeness.

Fig. 9 shows the distribution of needle visibility for insertion angle of $35^{\circ}$. In this figure, for fractional (not full) length i.e., $12 \mathrm{~mm}$, the local maxima are in the position of $25 \%, 55 \%$, and $78 \%$ of $12 \mathrm{~mm}$. While, for full length, i.e., $30 \mathrm{~mm}$, the local maxima are in the position of $17 \%, 45 \%$, and $82 \%$ of $30 \mathrm{~mm}$.
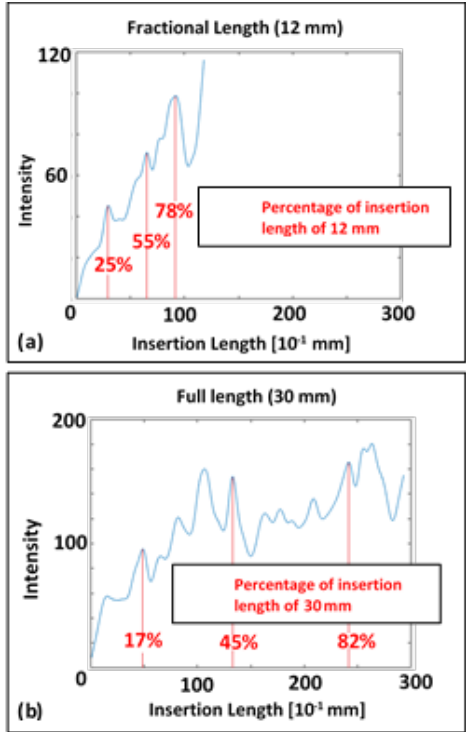

Fig. 9 the distribution of needle visibility for insertion angle of $35^{\circ}$ (linear transducer, $12 \mathrm{MHz}$ )

With one way ANOVA statistical analysis, for each insertion length, data of positions of local maxima in percentage of insertion length are compared between data group of fractional length and data group of full length. For example, Table 1 shows the data for insertion angle of $35^{\circ}$. Data group 1 (fractional length) are compared to data group 2 (full length). From this comparison, it is obtain that $\mathrm{F}_{\text {test }}=$ 0.04 , while $\mathrm{F}_{\text {statistics }}=7.71$. In this case, $\mathrm{F}_{\text {test }}<\mathrm{F}_{\text {statistics }}$, so that the result support null hypothesis $\mathrm{H}_{0}$, i.e., the variable of insertion length (fractional vs. full) does not significantly affect the pattern of visibility distribution along the needle.

Table 1. one-way ANOVA for data of insertion angle of $35^{\circ}$ (linear transducer, $12 \mathrm{MHz}$ )

\begin{tabular}{|l|l|l|}
\hline $\begin{array}{l}\text { Percentage of } \\
\text { insertion length }\end{array}$ & $\begin{array}{l}\text { Fractional } \\
\text { Length (12 } \\
\text { mm) }\end{array}$ & $\begin{array}{l}\text { Full } \\
\text { Length } \\
\mathbf{( 3 0} \mathbf{~ m m})\end{array}$ \\
\hline Local Maxima 1 & $25 \%$ & $17 \%$ \\
\hline Local Maxima 2 & $55 \%$ & $45 \%$ \\
\hline Local Maxima 3 & $78 \%$ & $82 \%$ \\
\hline
\end{tabular}

For linear transducer, from 8 different insertion angles, it is obtained that all $\mathrm{F}_{\text {test }}<\mathrm{F}_{\text {statistics. }}$. The results suggest that for all insertion angles, the distributions of needle visibility have similar pattern and they are not affected by the insertion length. 
For curved transducer, the insertion angles are divided into 11 angles within the range of $50^{\circ}-70^{\circ}$. For each insertion angle, the insertion length is classified into 3 length, i.e., full insertion length and two fractional (not full) insertion lengths. The position percentage of local maxima and local minima to the total length of the needle will be analyzed with statistical analysis to evaluate their likeness.

Fig. 10 shows the distribution of needle visibility for insertion angle of $60^{\circ}$. In this figure, for the first fractional (not full) length i.e., $19 \mathrm{~mm}$ (Fig. 10 (a)), the local maxima are in the position of $8 \%, 46 \%$, and $73 \%$ of $19 \mathrm{~mm}$. For the second fractional length i.e., $32 \mathrm{~mm}$ (Fig. 10 (b)), the local maxima are in the position of $3 \%, 33 \%$, and $84 \%$ of $32 \mathrm{~mm}$. While, for full length (Fig. 10 (c)), i.e., $37 \mathrm{~mm}$, the local maxima are in the position of $5 \%, 31 \%$, and $70 \%$ of $37 \mathrm{~mm}$.

With one way ANOVA statistical analysis, for each insertion length, data of positions of local maxima in percentage of insertion length are compared between data group of fractional length and data group of full length. For example, Table 2 shows the data for insertion angle of $60^{\circ}$. Data group 1 (fractional length I) are compared to data group 2 (fractional length II) and data group 3 (full length). From this comparison, it is obtain that $\mathrm{F}_{\text {test }}=0.03$, while $\mathrm{F}_{\text {statistics }}=5.14$. In this case, $\mathrm{F}_{\text {test }}<\mathrm{F}_{\text {statistics, }}$, so that the result support null hypothesis $\mathrm{H}_{0}$, i.e., the variable of insertion length (fractional vs. full) does not significantly affect the pattern of visibility distribution along the needle.

Table 2. one-way ANOVA for data of insertion angle of $60^{\circ}$ (curved transducer, $6 \mathrm{MHz}$ )

\begin{tabular}{|l|l|l|l|}
\hline $\begin{array}{l}\text { Percentage of } \\
\text { insertion length }\end{array}$ & $\begin{array}{l}\text { Fractional } \\
\text { Length I } \\
\mathbf{( 1 9} \text { mm) }\end{array}$ & $\begin{array}{l}\text { Fractional } \\
\text { Length II } \\
\text { (32 } \mathbf{~ m m})\end{array}$ & $\begin{array}{l}\text { Full } \\
\text { Length } \\
\text { (37 mm) }\end{array}$ \\
\hline Local Maxima 1 & $8 \%$ & $3 \%$ & $5 \%$ \\
\hline Local Maxima 2 & $46 \%$ & $33 \%$ & $31 \%$ \\
\hline Local Maxima 3 & $73 \%$ & $84 \%$ & $70 \%$ \\
\hline
\end{tabular}

For curved transducer, from 8 different insertion angles, it is obtained that all $F_{\text {test }}<\mathrm{F}_{\text {statistics. }}$. The results suggest that for all insertion angles, the distributions of needle visibility have similar pattern and they are not affected by the insertion length.
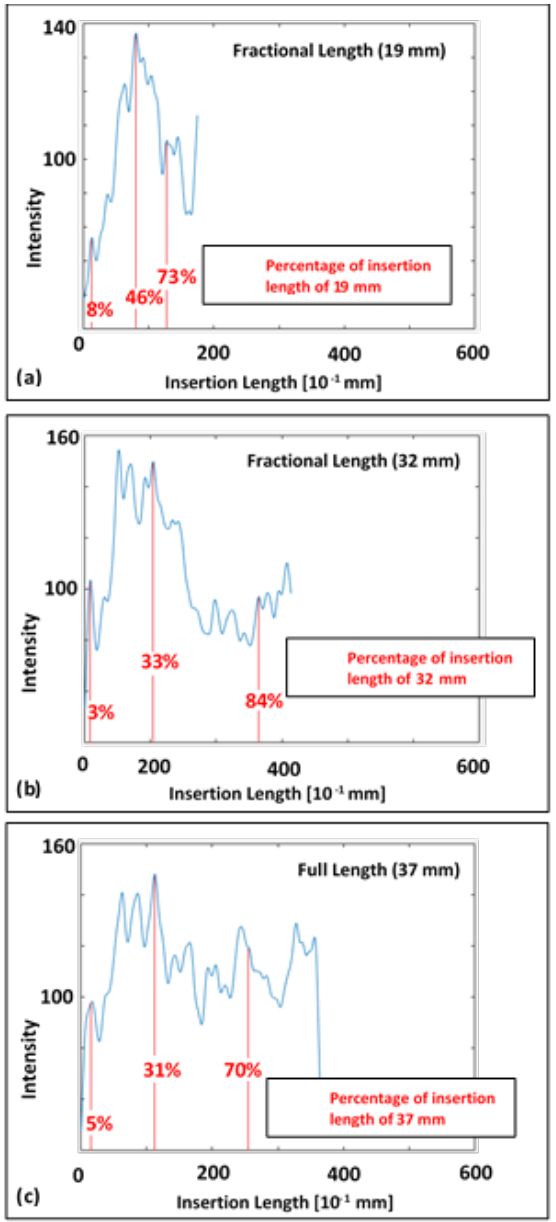

Fig. 10 the distribution of needle visibility for insertion angle of $60^{\circ}$ (curved transducer, $6 \mathrm{MHz}$ )

\section{B. The Visibility Map}

The visibility map will be evaluated for 2 type of transducers, i.e., linear transducer $(12 \mathrm{MHz})$ and curved transducer $(6 \mathrm{MHz})$. This visibility pattern is described by colors representing intensity level of B-mode image, where good visibility is represented by yellowish to reddish color and poor visibility is represented by blueish color approaching background color. The intensity level represents gray-scales in 8-bit.

\section{The Visibility Map for Linear Transducer (12 MHz)}

The visibility map for linear transducer is shown in Fig. 11. Observed from Fig. 11, for small insertion angle $\left(27^{\circ}-30^{\circ}\right)$, the needle has poor visibility at the beginning part of the shaft, then it gradually tends to have good visibility at the middle part and the tip. For steeper insertion angle $\left(30^{\circ}-60^{\circ}\right)$, the needle has good visibility at the beginning part of the shaft, and slightly decrease at the middle part, then it tends to increase again towards the tip. 


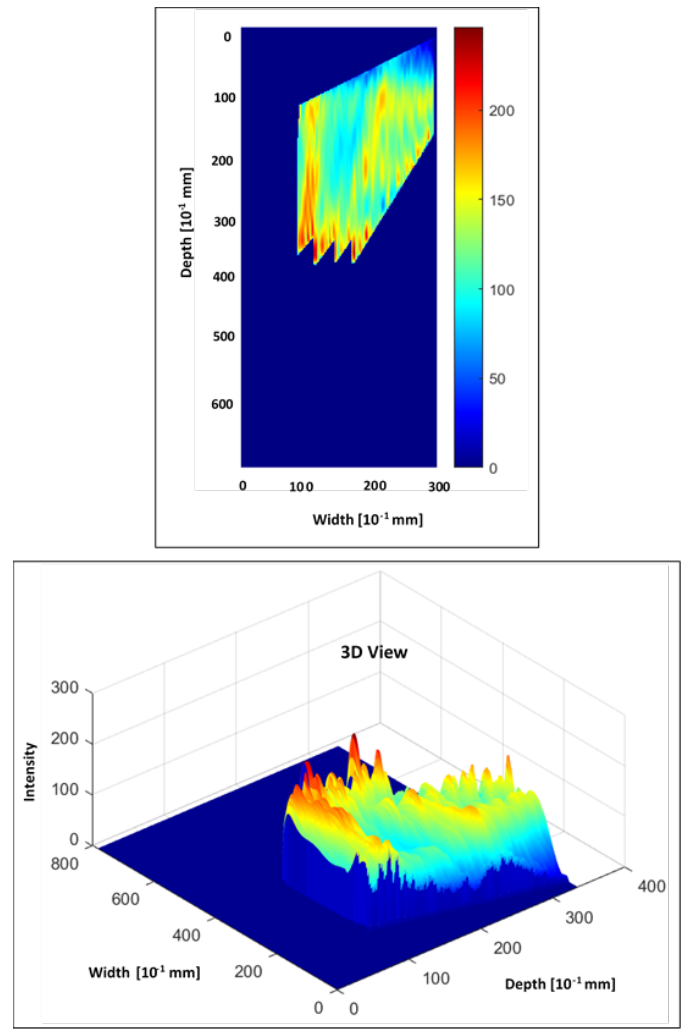

Fig. 11 the visibility map (linear transducer, $12 \mathrm{MHz}$ )

Evaluated from the whole area of insertion in Fig. 11, the needle has tendency to have good visibility at the part towards the tip even though the tip is excluding from the region of interest (ROI). This is caused by the comet tail artifact at the tip due to reverberation or multiple reflections when the ultrasound wave hits a strong reflecting object. That object has relatively large difference of acoustic impedance with its surrounding medium.

Then, the visibility map in Fig. 11 is cropped into a trianglelike segment in Fig. 12. In this figure, the map is cropped between insertion angle of $30^{\circ}$ and $45^{\circ}$. The triangle-like segment within this focused range can be used to predict the visibility pattern more precisely within narrower area $\left(30^{\circ}\right.$ $45^{\circ}$ ) of the total insertion area.

Furthermore, the triangle-like segment from Fig. 12 is converted into intensity graph in Fig. 13 to predict the distribution of needle visibility within those range $\left(30^{\circ}, 37.5^{\circ}\right.$, $\left.45^{\circ}\right)$. In this case, the patterns of visibility within the range of $30^{\circ}-45^{\circ}$ are relative similar with one another.
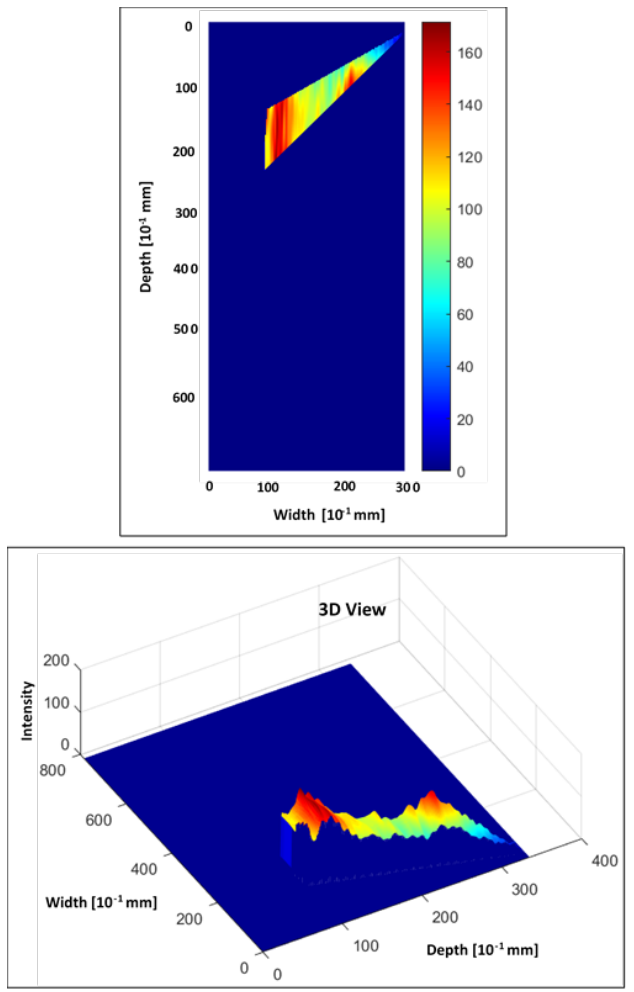

Fig. 12 the triangle-like segment cropped from the visibility map within the insertion angle of $35^{\circ}-45^{\circ}$ (linear transducer, $12 \mathrm{MHz}$ )
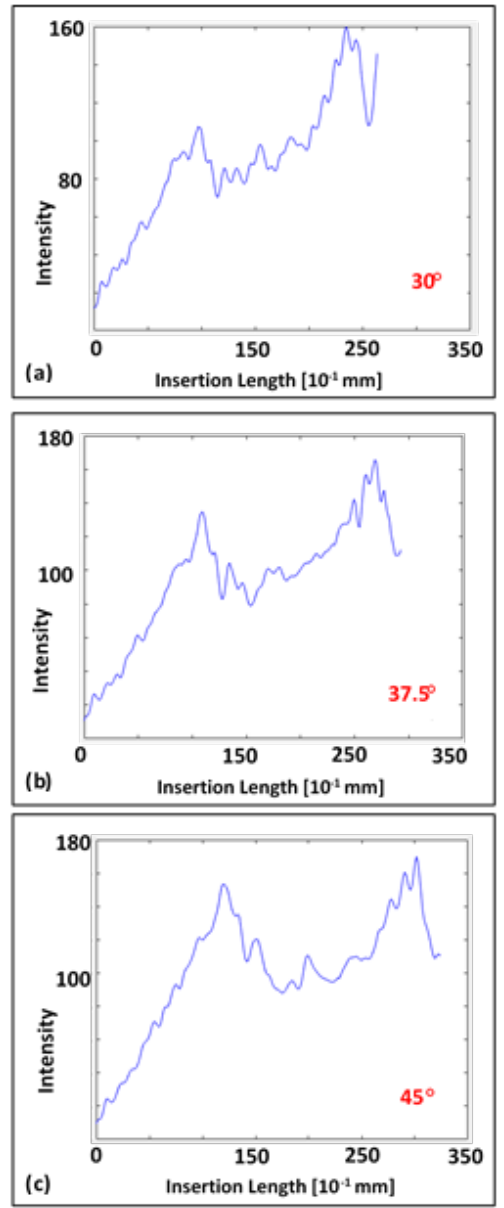

Fig. 13 the predicted distribution of needle visibility within the insertion angle of $35^{\circ}-45^{\circ}$ (linear transducer, $12 \mathrm{MHz}$ ) 


\section{The Visibility Map for Curved Transducer (6 MHz)}

The visibility map for curved transducer is shown in Fig. 14. Observed from Fig. 14, generally, the whole area of insertion can be divided into 5 areas based on the insertion length. The needle has good visibility at the first beginning (the $1^{\text {st }} 20 \%$ of full insertion length) and towards the tip (the $5^{\text {th }} 20 \%$ of full insertion length). The best visibility is shown at the $3^{\text {rd }} 20 \%$ of full insertion length. Aside from those areas, the visibility tends to decrease.

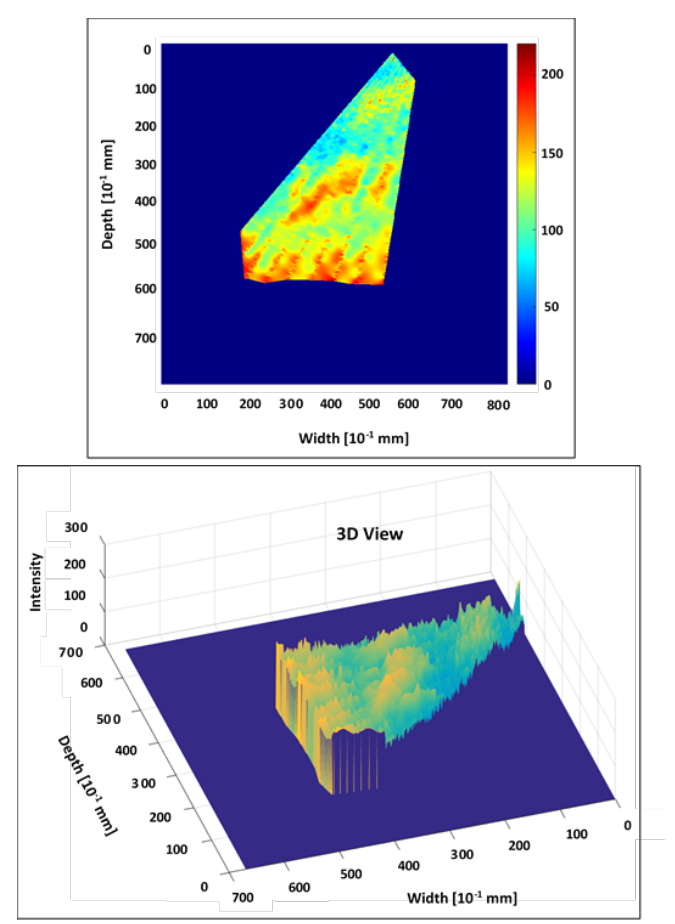

Fig. 14 the visibility map (curved transducer, $6 \mathrm{MHz}$ )

Evaluated from the whole area of insertion in Fig. 14, the needle has tendency to have good visibility at the part towards the tip even though the tip is excluding from the region of interest (ROI). This is caused by the comet tail artifact at the tip due to reverberation or multiple reflections when the ultrasound wave hits a strong reflecting object. That object has relatively large difference of acoustic impedance with its surrounding medium.

Then, the visibility map in Fig. 14 is cropped into a trianglelike segment in Fig. 15. In this figure, the map is cropped between insertion angle of $65^{\circ}$ and $75^{\circ}$. The triangle-like segment within this focused range can be used to predict the visibility pattern more precisely within narrower area $\left(65^{\circ}\right.$ $75^{\circ}$ ) of the total insertion area.

Furthermore, the triangle-like segment from Fig. 15 is converted into intensity graph in Fig. 16 to predict the distribution of needle visibility within those range $\left(65^{\circ}, 70^{\circ}\right.$, $75^{\circ}$ ). In this case, the patterns of visibility within the range of $65^{\circ}-75^{\circ}$ are relative similar with one another.

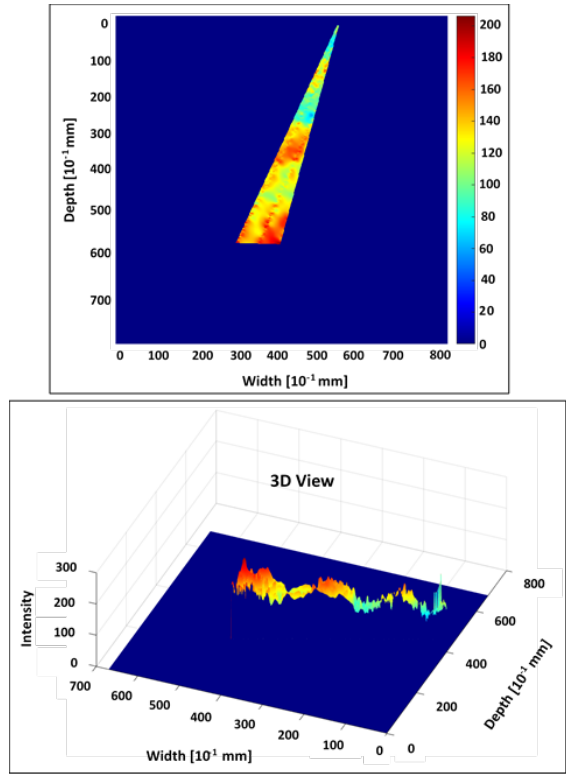

Fig. 15 the triangle-like segment cropped from the visibility map within the insertion angle of $65^{\circ}-70^{\circ}$ (curved transducer, $6 \mathrm{MHz}$ )
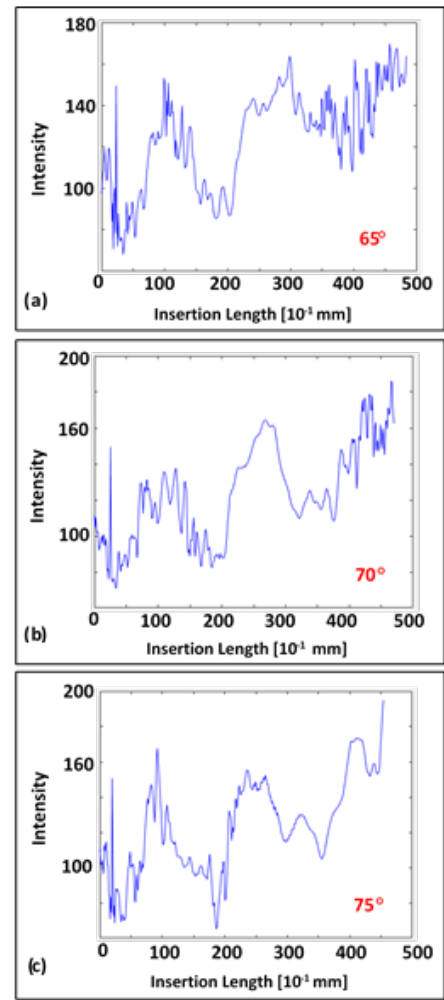

Fig. 16 the predicted distribution of needle visibility within the insertion angle of $65^{\circ}-70^{\circ}$ (curved transducer, $6 \mathrm{MHz}$ )

\section{CONCLUSION}

The experiment of needle insertion in this research is conducted to evaluate the significant influence of certain technical factor in needle insertion to the needle visibility, e.g., the effect of insertion length to the distribution of needle visibility along the shaft of the needle. In addition to that, the experiment is also performed to develop the leveling system of needle visibility. This development is very important as an 
initial step before further investigation about fundamental physics behind needle visibility and eventually further development of needle visibility enhancement.

Further research about investigation of physical phenomena that underlays needle visibility and needle visibility enhancement cannot be done without this initial approach to evaluate needle visibility comprehensively. The information about the trend of key technical parameters that influence needle visibility can be the starting points to investigate the candidate parameters that can be re-engineered to enhance needle visibility.

Apart from that, the graph of visibility distribution and the visibility map can be used practically to predict the distribution of needle visibility in a specific case of needle insertion in clinical application. Since, the pattern of needle visibility shown in the visibility map is unique for each insertion case, it can be used as a specific reference for a physician limited by the specification of needle and ultrasound system, i.e., the range of transducer's frequency and the needle's size. The clinical application of needle insertion procedure can be applied in regional anesthesia (spinal or epidural) and biopsy. Visual feedback in the form of needle Bmode ultrasonography can be very helpful to improve the accuracy of needle positioning and avoid needle insertion errors.

The results suggest that both with linear and curved transducer, for all insertion angles, the distributions of needle visibility have similar pattern and they are not affected by the insertion length.

\section{ACKNOWLEDGMENT}

We acknowledge Indonesia Endowment Fund for Education (LPDP) for providing scholarship for this doctoral research and also The Ministry of Research, Technology and Higher Education of Republic of Indonesia for competency-based research funding.

\section{REFERENCES}

[1] A. Naaji, M-C. Popescu, G. C. Sarla, "Software filtering applications for the analysis of dental images," WSEAS Transactions on Signal Processing, vol. 16, pp. 59-69, 2020.

[2] H. Susanti, Suprijanto, D. Kurniadi, "Two-dimensional mapping of needle visibility with linear and curved array for ultrasound-guided interventional procedure," in Proc. AIP Conference, vol. 1933, Bali, 2017, pp. 040004-1-4..

[3] K. J. Chin, A. Perlas, V. W. S. Chan, R. Brull, "Needle visualization in ultrasound-guided regional anesthesia: challenges and solutions," Regional Anesthesia and Pain Medicine, vol. 33, no. 6, pp. 532-544, Nov. 2008

[4] P. Marhofer, M. Greher, S. Kapral, "Ultrasound guidance in regional anaesthesia," British J Anaesth, vol. 94, pp. 7-17, Jan. 2005.

[5] S. Dencks, H. Susanti, G. Schmitz, "Needle visibility for deep punctures with curved arrays," in Proc. IEEE International Ultrasonics Symposium, Chicago, 2014, pp. 1880-1883.

[6] S. Guo, A. Schwab, G. McLeod, G. Corner, S. Cochran, R. Eisma, R. Soames, "Echogenic regional anaesthesia needles: a comparison study in thiel cadavers," Ultrasound in Medicine and Biology, vol. 38, pp. 702-707, Apr. 2012.
[7] S. Hebard, G. Hocking, K. Murray, "Two-dimensional mapping to assess direction and magnitude of needle tip error in ultrasound-guided regional anaesthesia," Anaesthesia and Intensive Care Journal, vol. 39, pp. 1076-1081, Nov. 2011.

[8] S. Karstrup, J. Brøns, L. Morsel, N. Juul, P. von der Recke, "Optimal set-up for ultrasound guided punctures using new scanner applications: an in-vitro study," European Journal of Ultrasound, vol. 15, pp. 77-84, Jun. 2002.

[9] T. L. de Jong, N. J. van de Berg, L. Tas, J. Dankelman, J. J. van den Dobbelsteen, "Needle placement errors. Do we need steerable needles in interventional radiology?," Medical Devices: Evidence and Research, vol. 11, pp. 259-265, Aug. 2018.

[10] T. Maecken, M. Zenz, T. Grau, "Ultrasound characteristics of needles for regional anesthesia," Regional Anesthesia and Pain Medicine, vol. 32, no. 5, pp. 440-447, Sept. 2007.

[11] W. C. Culp, T. C. McCowan, T. C. Goertzen, T. G. Habbe, M. M. Hummel, R. F. LeVeen, J. C. Anderson, "Relative ultrasonographic echogenicity of standard, dimpled, and polymeric-coated needles," $J$ Vasc Interv Radiol, vol. 11, pp. 351-358, Mar. 2000. 